REDES- Revista hispana para el análisis de redes sociales

Vol.9\#1, Dic. 2005

http://revista-redes.rediris.es

\title{
Pluralidad teórica, metodológica y técnica en el abordaje de las redes sociales: hacia la "hibridación" disciplinaria
}

\author{
Estrella Gualda Caballero - Universidad de Huelva ${ }^{1}$
}

\begin{abstract}
RESUMEN
Discutiendo sobre algunos trabajos que se presentan en el volumen noveno de la Revista Redes. Revista Hispana para el análisis de las redes sociales, este artículo incorpora un conjunto de reflexiones sobre el análisis de las redes sociales argumentando que esta específica área de análisis se encuentra en un cruce de caminos interdisciplinarios que vienen aportando recientemente ideas adicionales para la comprensión de las redes sociales y del funcionamiento de diversas parcelas de la sociedad. Vistas las aportaciones en su conjunto, e incluso analizando algunas particularmente, pueden entenderse éstas como respuestas plurales (en lo teórico, metodológico y técnico) a preguntas relevantes en el ámbito de las ciencias sociales (y otras) que amplían nuestra comprensión del mundo y generan nuevas incógnitas sobre las que seguir trabajando. En la medida en que estas contribuciones combinan ideas, procedimientos, etc. de diversas ciencias que se ponen al servicio del ARS, puede hablarse de que en este campo se está practicando una productiva hibridación disciplinaria.
\end{abstract}

\section{PALABRAS CLAVES}

Redes sociales. Hibridación disciplinaria. Pluralidad teórica, metodológica y técnica.

\begin{abstract}
Discussing on some works that have been presented in the nineth volume of the Journal Redes. Revista Hispana para el análisis de las redes sociales, this article incorporates a group of reflections on interesting questions today in the analysis of social networks, arguing that this specific area of analysis is established in a crossing of interdisciplinary roads that contributes recently with additional ideas for the comprehension of the social networks and the way of functioning of some parts of our society. If we analyse the contributions in a global perspective, and even analyzing some of them particularly, they can be understood as plural answers (in a theoretical, methodological and technical point of view) to prominent questions in the social sciences and others, and they expand our comprehension of the social world. They also suggest new questions to carry on with the reflections. Combining ideas, procedures, etc. of diverse sciences at the service of the Social Network Analysis could be understood as no more than practising a productive disciplinary hybridization with interesting contributions.
\end{abstract}

\section{KEYWORDS}

Social Networks. Disciplinary hybridization. Theoretical, methodological and technical plurality.

\footnotetext{
${ }^{1}$ Enviar correspondencia a: Estrella Gualda, estrella@uhu.es; equalda@ono.com.
} 


\section{Presentación}

Del 7 al 9 de septiembre de 2005 tuvo lugar en Sevilla el I International Workshop de la Red Hispana para el Análisis de Redes Sociales, con el apoyo logístico de la Revista Redes. Revista Hispana para el análisis de las redes sociales y el Centro de Estudios Andaluces, que cedió sus espacios para la celebración del evento. Éste contó también con el patrocinio del Ministerio de Educación y Ciencia y el Ministerio de Asuntos Exteriores y de Cooperación (Agencia Española de Cooperación Internacional), que financiaron la estancia de investigadores externos especializados en el análisis de las redes sociales. La organización corrió a cargo de los profesores José Luis Molina, del Dpto. de Antropología Social y Cultural de la Universidad Autónoma de Barcelona; de Isidro Maya, de la Universidad de Sevilla y de Estrella Gualda, de la Universidad de Huelva. Este Workshop se configuró igualmente como III Seminario para el análisis de Redes Sociales en Sevilla, continuando el trabajo realizado en el II Seminario para el análisis de redes sociales en Sevilla que tuvo lugar entre el 2 y 3 de diciembre de 2004, y se centró en la temática de "Redes personales y comunidades inmigradas", patrocinado en esa ocasión por el Centro de Estudios Andaluces, la Revista Redes y el Laboratorio de Redes Personales y Comunidades (LRPC). Rasgos comunes de estos Seminarios fueron su carácter internacional, si pensamos en los investigadores asistentes, y su orientación interdisciplinaria, por cuanto interesados en el análisis científico de las redes sociales, se reunieron especialistas de campos disciplinares diversos, circunscritos a las ciencias sociales y humanas, entre otras.

Este artículo, considerando algunos de los trabajos que allí se presentaron y que se publican en el volumen noveno de la Revista Redes, así como una parte de las discusiones interdisciplinares internacionales que están teniendo lugar en el ámbito temático del análisis de las redes sociales (ARS a partir de aquí), presenta, desde una lectura personal, cuáles son algunas de las fortalezas y aportaciones que éste proporciona al avance del conocimiento científico, sin perder de vista algunas de sus limitaciones.

\section{Pluralidad teórica: herencias y preocupaciones disciplinares}

Aunque el campo del ARS se ha configurado como un espacio de encuentro interdisciplinar y de enriquecimiento de las investigaciones que incorporan esta perspectiva de análisis, no puede negarse que las herencias disciplinares tienen gran peso en la configuración de las investigaciones que se realizan particularmente, así como en los objetivos, objetos y sujetos de dichas indagaciones. Esto, por otra parte, parece lógico, pues cada investigador se nutre 
de orientaciones concretas, aunque puedan ser varias y distintas entre sí según su propia biografía personal, relacional y académica. Consciente o inconscientemente, intuyo, entre las aproximaciones de algunos trabajos centrados en el ARS encontramos tanto estrategias ${ }^{2}$ del estilo a una "inmersión a fondo" en el análisis de redes sociales, diluyéndose y perdiéndose de alguna forma el campo disciplinar propio en un conjunto pluralmente compuesto por aportaciones de múltiples campos, como otras en las que, desde el bagaje proporcionado por disciplinas concretas, el investigador incorpora el ARS, como aspecto complementario, pero no exclusivo, que ocupa un lugar más o menos importante, en su diseño y práctica investigadora, en aras de una mejor descripción y conocimiento de su objeto de interés.

En cualquier caso, el ARS globalmente se desarrolla y aporta elementos al conocimiento de la realidad social, independientemente de la perspectiva particular de investigaciones concretas. Este abordaje interdisciplinario con mayores orientaciones hacia campos concretos, o esas aproximaciones con mayor inmersión en el ARS, pueden ser observadas en la lectura de algunos artículos publicados en algunas de las revistas internacionales actualmente centradas en la cuestión: Social Networks (http://www1.elsevier.com/homepage/sae/son/), Journal of Social Structure (http://www.cmu.edu/joss/), Connections, Redes. Revista hispana para el análisis de las redes sociales (http://revista-redes.rediris.es).

En este punto, no deja de ser expresivo el incremento exponencial de los trabajos publicados en el mundo cuyo eje de interés gira en torno a las "redes sociales", como se rastrea fácilmente accediendo al Social Science Citation Index o en cualquier otra base de datos especializada (véase, por ejemplo, en Sociological Abstract). Las publicaciones en este campo temático no han dejado de crecer $y$, como apunta Freeman (2004), es interesante observar, igualmente, que las que abordan con interés la influencia o participación de las "redes sociales" no se circunscriben a revistas especializadas temáticamente como las citadas arriba, sino que se dispersan entre un variopinto y extenso listado de revistas científicas de diversas ramas de saber.

Sin pretender llevar las afirmaciones al ámbito de la generalización, sí puede defenderse que los trabajos presentados en el volumen noveno de la Revista Redes se impregnan de orientaciones disciplinares ligadas a la biografía de sus autores, sin agotarse en ellas. Veremos seguidamente que se aprecian éstas tanto respecto

\footnotetext{
${ }^{2}$ Que se concretan finalmente en los resultados de investigación.
} 
a algunos marcos u orientaciones teóricas de partida, como respecto a las metodologías que se emplean, así como a las conclusiones que se formulan, orientadas por objetivos notablemente diferentes.

\section{a) Redes sociales en la actividad textil (Teves, 2005)}

El caso de Laura Teves, de la Universidad Nacional de la Plata, si bien se sitúa en una perspectiva de la antropología sociocultural, sugiere igualmente una aproximación a elementos de análisis organizacional y grupal en economías que tienen elementos locales y componentes globales. Su título "Análisis de Redes sociales y actividades económicas en las comunidades de Molinos" es por sí mismo expresivo de que el análisis conjuga esta última dimensión. Se trata de un trabajo que al estudiar la red de relaciones del ego permite observar que estas relaciones van más allá la unidad doméstica, con lo que se introduce una interesante línea de abordaje con paradas en elementos micro, meso y macro de la actividad textil. Al mismo tiempo, se discute sobre el hecho de que las decisiones individuales no se producen en el vacío, sino que tienen que ver con la organización intragrupal. El vínculo descubierto de transacciones económicas cotidianas en la actividad textil muestra a su vez "la complementariedad ecológica de los microambientes andinos" y la conectividad de la red, hasta el punto de que si se suprimen algunos nodos, la red puede quedar desconectada, lo que permite hablar a la autora de su cohesión social. De hecho, la red descrita contempla los puntos más importantes de la misma, que coinciden en ser los lugares que más recursos naturales tienen. No puede olvidarse en su trabajo que la población molinista recorre cerros y valles con motivo de la actividad textil, y al hacerlo, se visitan unidades domésticas y se establecen relaciones sociales de amistad, parentesco y comerciales que funcionan a modo de "circuito". También se descubren tres rutas diferentes de circulación de recursos.

\section{b) Calidad de vida en salud y desplazados por violencia política (Palacio y Madariaga, 2005)}

Una segunda línea de análisis, esta vez orientada hacia la psicología, es la que se presenta en el trabajo de Jorge Palacio y Camilo Madariaga, investigadores de la Universidad del Norte en Colombia, titulado "Redes sociales personales y calidad de vida en personas desplazadas por violencia política: el caso de Barranquilla (Colombia)". En este caso, más que desde una aproximación antropológica, como se traducía en el trabajo reseñado previamente, se enfoca el estudio de las redes personales de los desplazados con el interés de conocer cómo 
afecta dicha condición de "desplazado por violencia política" en su proceso particular de adaptación y, concretamente, en su calidad de vida en salud. La línea argumental intenta perseguir la influencia de las redes sociales del asentamiento de desplazados estudiado en la satisfacción subjetiva medida a través de variables de calidad de vida. Se contextualiza teóricamente el análisis contemplando aspectos como la fragmentación familiar, los sentimientos de impotencia, la tristeza, la ansiedad, la depresión, el estrés o la presión psicológica, pero también se discute sobre los conflictos con los residentes y la importancia de generar sentido de comunidad para lograr procesos de adaptación más exitosos.

\section{c) Identificaciones culturales (Aguilar, 2005)}

El trabajo de Claudia Aguilar, del Departamento de Antropología Social y Cultural de la Universidad Autónoma de Barcelona, centrado en la "Visualización de redes personales en Sarajevo", aunque no exclusivamente, se nutre en gran medida de la antropología, haciendo foco de su interés para la visualización de las redes elementos como las identidades e identificaciones culturales. Identidades que en este trabajo pueden tomar una vertiente nacionalista y religiosa. De un lado se describirían, atendiendo al discurso oficial en Bosnia y Herzegovina ( $\mathrm{BiH})$, las diferencias que la población en Sarajevo percibe entre las categorías de "serbio", "croata" y "bosníaco o musulmán" (como categoría mixta: nacional-religiosa). Por otro, se hacen distinciones como las de "ateo", o "musulmán", por ejemplo. Las metas de esta descripción son referidas al interés de conocer la complejidad que rodea el "proceso de identificación", lo que se hace bajo los presupuestos de la existencia de identificaciones múltiples (donde juegan desde autoidentificaciones étnicas, hasta la percepción de las identificaciones que hacen otros de uno mismo), con la argumentación de fondo de que los procesos de identificación son de carácter relacional, en constante negociación.

\section{d) Grupos y organizaciones, redes sociales y capital social (Forni y Nardone, 2005)}

Pablo Forni y Mariana Nardone, del Instituto de Investigación en Ciencias Sociales de la Universidad del Salvador, Argentina, presentan una investigación sobre "Grupos solidarios de microcrédito y redes sociales: sus implicancias en la generación de capital social en barrios del Gran Buenos Aires". En este trabajo se concreta una mirada sociológica que pone su énfasis en la existencia y construcción de grupos solidarios y organizaciones sociales. En torno a estos grupos y organizaciones se deja notar la actividad de las redes sociales. Otro eje de interés 
es cómo éstos se estructuran y relacionan entre sí a lo largo del tiempo generando capital social. Capital social que, por su carácter, puede ser bonding social capital o capital social de unión (un tipo de capital social intensivo, donde el afecto y la emoción, así como la proximidad de la familia y los amigos es nota característica); linking social capital o capital social de vinculación; y bridging social capital o capital social de aproximación, capital social que sirve de puente.

\section{e) Visualización de redes sociales (Brandes, Kenis y Raab, 2005)}

El trabajo de Brandes, Kenis y Raab, titulado "Explanation Through Network Visualization", tiene un carácter diferente al de los autores anteriores pues si bien se toman dos casos como ejemplos para la argumentación, la meta de fondo es presentar razonamientos que den valor a la "visualización" como vía de obtener la explicación de fenómenos estudiados científicamente. La coherencia disciplinar habría que encontrarla ahora en un grupo de trabajo dividido entre personas del Department of Organisation Studies de la Tilburg University, de Holanda y el Department of Computer \& Information Science, de la University of Konstanz, de Alemania. Esta mayor aproximación a la informática se deja ver a través de la creación del programa visone (http://www.visone.info/), que es empleado para producir la visualización de redes sociales en los términos de las investigaciones tomadas de ejemplo. Una de ellas, tiene que ver con medidas preventivas del Sida para usuarios de drogas y el otro es un estudio sobre la privatización de industrias navales y del acero en el este de Alemania durante su transformación económica posterior a los noventa.

\section{F) Redes sociales y felicidad en la población mayor (Gualda, 2005)}

El último trabajo que se reseña fue presentado igualmente en el $I$ International Workshop de la Red Hispana para el Análisis de Redes Sociales, bajo el título de Redes sociales y personales y su implicación en los estados de ánimo y percepciones de felicidad de personas mayores. Estudio de casos en Berrocal y Villanueva de las Cruces (Huelva) (Gualda, 2005b). Algunos análisis de este texto (que no se incorpora como artículo al volumen), junto a elementos elaborados posteriormente en el contexto de esta investigación (Gualda, 2005a), los empleamos en este trabajo con el fin de incorporar ideas complementarias y nuevas líneas argumentales en este artículo. El eje central de la discusión tiene que ver con el intento de delimitar las estructuras y tipologías de las redes sociales 
localizadas en la población mayor, de acuerdo a sus autodeclaraciones respecto a su estado de felicidad. La aproximación se hace desde una sociología pluralista en lo teórico y metodológico.

\section{G) Otros temas, otros enfoques}

Qué duda cabe de que el volumen noveno de la revista Redes es un buen ejemplo del actual quehacer interdisciplinar en este campo del saber, que se enriquece día a día con las aportaciones de ciencias que se han implicado en el ARS, sean éstas sociales, humanas, técnicas, naturales u otras. Junto al abanico temático aquí esbozado, habría que citar otros muchos, siendo un botón de muestra estos trabajos que han sido publicados por la misma Revista Redes. Revista Hispana para el Análisis de las Redes Sociales. Recuérdese con Freeman (2004) el intenso crecimiento en las últimas décadas de las publicaciones centradas en el ARS.

Por otra parte, desde la óptica de las Ciencias Sociales, el hecho de fortalecer y diversificar nuestros conocimientos teóricos introduciendo elementos propios de otros campos disciplinarios no es tarea nueva pues ya desde los propios orígenes de estas disciplinas, por ejemplo de la Sociología (citando un ejemplo próximo), puede rastrearse dicha circunstancia, que habría que trasladar a su vez a momentos más remotos, en los que los saberes sobre el mundo no se encontraban tan "académicamente" compartimentalizados.

Si bien actualmente en algunos ámbitos del saber parece que se tiende hacia la hiperespecialización temática y técnica, lo que se concreta en las Ciencias Sociales no suele ser esta "ortodoxia" o "pureza disciplinaria" que tiene poco sentido para la comprensión de fenómenos sociales complejos, aunque por otra parte, y al menos en el contexto español, la "academia" se encuentre artificial, administrativa y funcionalmente compartimentalizada y fragmentada en ciencias 0 disciplinas diferentes. Una lógica académica que en muchos momentos no es consonante con la lógica y práctica investigadora, más pragmática, en el sentido de que rompe o supera las barreras disciplinarias para acometer temas complejos desde una complementariedad disciplinaria que, por otra parte, no es ajena. No debe olvidarse que la formación académica universitaria en estas disciplinas suele estar compuesta por curriculums formativos igualmente complejos. EI ARS se sitúa en este escenario de hibridación disciplinaria. 


\section{Metodología, técnica y software: diferentes momentos en la incorporación del ARS}

Las aportaciones del análisis de redes sociales permiten observar igualmente cómo diferentes tradiciones disciplinares incorporan sus bagajes metodológicos y técnicos en alguna fase de una investigación que añade entre sus etapas el ARS. Así, por ejemplo, lo específico y común de gran parte de las investigaciones que pretenden conocer cómo es la estructuración en redes sociales de alguna comunidad o grupo concreto, qué personas mantienen relaciones entre sí, o qué colectivos o instituciones se encuentran vinculadas (según el enfoque sea más individual o colectivo), suele ser que incorporan en su trabajo de campo una batería de preguntas que indagan de alguna forma sobre las redes. Esta batería de preguntas puede estar más o menos estandarizada, estructurada y probada. En este volumen hemos encontrado ambas aproximaciones (Cuadro 1).

Por otra parte, del mismo modo que encontramos orientaciones teóricas diferentes, algo equivalente ocurre respecto a las orientaciones metodológicas y técnicas, a saber, de carácter plural. No obstante, debe indicarse que, salvo por la incorporación de elementos específicos que tienen que ver con el análisis de redes sociales, las investigaciones que se incluyen en el volumen noveno de Redes se caracterizan por combinar o complementar esta aproximación hacia las redes sociales con estrategias metodológicas y técnicas de sus propias orientaciones disciplinares. 
ORIENTACIONES METODOLÓGICAS, TÉCNICAS Y SOFTWARE EN EL ANÁLISIS DE LAS REDES SOCIALES (VOL. 9 DE REDES) I

\begin{tabular}{|c|c|c|c|c|c|}
\hline Fase & Teves & $\begin{array}{c}\text { Palacio y } \\
\text { Madariaga }\end{array}$ & Aguilar & $\begin{array}{c}\text { Forni y } \\
\text { Nardone }\end{array}$ & Gualda \\
\hline $\begin{array}{l}\text { Aproximación } \\
\text { disciplinar }\end{array}$ & Antropología & Psicología & Antropología & Sociología & Sociología \\
\hline $\begin{array}{l}\text { Universo u } \\
\text { objeto de } \\
\text { estudio }\end{array}$ & $\begin{array}{c}\text { Redes sociales } \\
\text { en la actividad } \\
\text { económica }\end{array}$ & $\begin{array}{l}\text { Calidad de vida } \\
\text { y redes sociales } \\
\text { en desplazados }\end{array}$ & $\begin{array}{c}\text { Redes sociales } \\
\text { de jóvenes e } \\
\text { identificación } \\
\text { social } \\
\end{array}$ & $\begin{array}{l}\text { Grupos de } \\
\text { microcrédito }\end{array}$ & $\begin{array}{c}\text { Calidad de vida y } \\
\text { redes sociales en } \\
\text { población de } 55 \text { y } \\
\text { más años }\end{array}$ \\
\hline $\begin{array}{l}\text { Ámbito de } \\
\text { trabajo }\end{array}$ & $\begin{array}{c}\text { Valle cachalquí } \\
\text { salteño } \\
\text { (Argentina) }\end{array}$ & $\begin{array}{c}\text { Pinar del Río } \\
\text { (Colombia). } \\
\text { Asentamiento } \\
\text { en la periferia } \\
\text { noroeste de } \\
\text { Barranquilla }\end{array}$ & Sarajevo & $\begin{array}{c}\text { Cuartel V, } \\
\text { Moreno (Gran } \\
\text { Buenos Aires, } \\
\text { Argentina) }\end{array}$ & $\begin{array}{c}\text { Municipio rural de } \\
\text { Berrocal (Huelva, } \\
\text { España) }\end{array}$ \\
\hline $\begin{array}{l}\text { Orientación } \\
\text { predominante }\end{array}$ & Cualitativa & Cuantitativa & Cualitativa & Cualitativa & $\begin{array}{c}\text { Cuantitativa y } \\
\text { cualitativa }\end{array}$ \\
\hline $\begin{array}{l}\text { Técnica de } \\
\text { recogida de } \\
\text { datos }\end{array}$ & $\begin{array}{c}\text { Etnografía, } \\
15 \text { entrevistas } \\
\text { semiestruc- } \\
\text { turadas a } \\
\text { informantes. } \\
\text { Datos } \\
\text { discursivos y } \\
\text { observacio- } \\
\text { nales }\end{array}$ & $\begin{array}{c}19 \text { entrevistas, } \\
\text { con el apoyo de } \\
\text { un líder } \\
\text { comunitario } \\
\text { para contactar a } \\
\text { las primeras } \\
\text { familias }\end{array}$ & $\begin{array}{l}\text { Observación } \\
\text { participante. } \\
\text { Análisis de } \\
\text { redes perso- } \\
\text { nales de } 17 \\
\text { casos } \\
\text { (entrevistas } \\
\text { en profun- } \\
\text { didad) }\end{array}$ & $\begin{array}{l}17 \text { entrevistas } \\
\text { en profundidad } \\
\text { semiestruc- } \\
\text { turadas } \\
\text { Observación no } \\
\text { participante. } \\
\text { Grupo focal }\end{array}$ & $\begin{array}{l}115 \text { entrevistas } \\
\text { (información } \\
\text { cuasi-censal, más } \\
\text { que muestral). } \\
\text { Observación } \\
\text { participante. } \\
\text { Grupos de } \\
\text { discusión. } \\
\text { Entrevistas a } \\
\text { familiares de } \\
\text { mayores. }\end{array}$ \\
\hline $\begin{array}{l}\text { Perfil } \\
\text { entrevistados o } \\
\text { participantes en } \\
\text { grupos }\end{array}$ & $\begin{array}{l}\text { "Expertos" en } \\
\text { el sector textil }\end{array}$ & $\begin{array}{c}\text { Adultos de } \\
\text { ambos sexos } \\
\text { con } 3 \text { meses ó } \\
\text { menos y } 1 \text { año } \\
\text { o más en el } \\
\text { asentamiento }\end{array}$ & Jóvenes & $\begin{array}{c}\text { Miembros de } \\
\text { colectivos } \\
\text { solidarios, } \\
\text { informantes } \\
\text { clave, directivos }\end{array}$ & $\begin{array}{l}\text { Mayores de } 55 \\
\text { años. Familiares } \\
\text { citados como } \\
\text { apoyo básico por } \\
\text { los mayores }\end{array}$ \\
\hline $\begin{array}{l}\text { Instrumentos } \\
\text { empleados para } \\
\text { conocer las } \\
\text { redes sociales }\end{array}$ & $\begin{array}{c}\text { Guión que } \\
\text { incorpora } \\
\text { preguntas } \\
\text { sobre redes } \\
\text { sociales en la } \\
\text { actividad textil }\end{array}$ & $\begin{array}{c}\text { Batería } \\
\text { específica } \\
\text { pretestada } \\
\text { sobre redes } \\
\text { sociales } \\
\text { (Barrero, 1980; } \\
\text { Arizona Social } \\
\text { Support } \\
\text { Interview } \\
\text { Schedule } \\
\text { (ASSIS) }\end{array}$ & $\begin{array}{l}\text { Cuestionario } \\
\text { diseñado } \\
\text { específica- } \\
\text { mente para } \\
\text { analizar las } \\
\text { redes sociales }\end{array}$ & $\begin{array}{c}\text { Guión } \\
\text { semiestruc- } \\
\text { turado de } \\
\text { cuestiones sobre } \\
\text { redes sociales }\end{array}$ & $\begin{array}{c}\text { Batería específica } \\
\text { pretestada sobre } \\
\text { redes sociales } \\
\text { (Barrero, 1980; } \\
\text { Arizona Social } \\
\text { Support Interview } \\
\text { Schedule (ASSIS) }\end{array}$ \\
\hline $\begin{array}{l}\text { Otros } \\
\text { instrumentos } \\
\text { para/en las } \\
\text { encuestas o } \\
\text { entrevistas }\end{array}$ & $\begin{array}{l}\text { Guiones } \\
\text { semiestruc- } \\
\text { turados }\end{array}$ & $\begin{array}{l}\text { Cuestionario } \\
\text { sobre Calidad de } \\
\text { Vida en Salud } \\
\text { (SF-36). Estudio } \\
\text { piloto para la } \\
\text { adaptación de } \\
\text { instrumentos }\end{array}$ & $\begin{array}{c}\text { Preguntas al } \\
\text { informante } \\
\text { sobre su } \\
\text { identificación y } \\
\text { otras } \\
\text { características } \\
\text { suyas y de las } \\
\text { personas } \\
\text { nombradas } \\
\end{array}$ & $\begin{array}{l}\text { Guión de } \\
\text { cuestiones } \\
\text { semiestruc- } \\
\text { turado }\end{array}$ & $\begin{array}{c}\text { Preguntas } \\
\text { sociodemográ- } \\
\text { ficas y otras } \\
\text { relativas a } \\
\text { características } \\
\text { familiares, de } \\
\text { salud, ocio y } \\
\text { tiempo libre, etc. } \\
\text { Pre-test. }\end{array}$ \\
\hline
\end{tabular}


ORIENTACIONES METODOLÓGICAS, TÉCNICAS Y SOFTWARE EN EL ANÁLISIS DE LAS REDES SOCIALES (VOL. 9 DE REDES) I (continuación)

\begin{tabular}{|c|c|c|c|c|c|}
\hline Fase & Teves & $\begin{array}{c}\text { Palacio y } \\
\text { Madariaga }\end{array}$ & Aguilar & $\begin{array}{c}\text { Forni y } \\
\text { Nardone }\end{array}$ & Gualda \\
\hline $\begin{array}{l}\text { Selección } \\
\text { muestral }\end{array}$ & Intencional. & $\begin{array}{l}\text { Intencional. } \\
\text { Bola de nieve }\end{array}$ & Intencional & $\begin{array}{l}\text { Intencional } \\
\text { (saturación) }\end{array}$ & $\begin{array}{c}\text { Cuasi-censal, } \\
\text { según datos del } \\
\text { Padrón }\end{array}$ \\
\hline $\begin{array}{l}\text { Criterios } \\
\text { muestrales }\end{array}$ & $\begin{array}{l}\text { "Experto" en la } \\
\text { actividad textil } \\
\text { en el valle } \\
\text { cachalquí } \\
\text { salteño }\end{array}$ & $\begin{array}{c}\text { Adultos, de } \\
\text { ambos sexos, } \\
\text { diferente } \\
\text { familia y que } \\
\text { habían llegado } \\
\text { al asentamien- } \\
\text { to hacía } \\
\text { menos de tres } \\
\text { meses y más } \\
\text { de un año }\end{array}$ & $\begin{array}{c}\text { Selección de } \\
\text { jóvenes que se } \\
\text { autoidenti- } \\
\text { fiquen con } \\
\text { categorías } \\
\text { culturales } \\
\text { diferentes } \\
\text { (nación, } \\
\text { religión) }\end{array}$ & $\begin{array}{c}\text { Según se } \\
\text { cumpliera o no } \\
\text { la garantía } \\
\text { solidaria; } \\
\text { diversificando } \\
\text { los grupos } \\
\text { según sus } \\
\text { vínculos } \\
\text { internos } \\
\text { (familiar, } \\
\text { vecinal, mixtos) }\end{array}$ & $\begin{array}{c}\text { Residente en } \\
\text { Berrocal, de } 55 \\
\text { y más años. } \\
\text { Sólo se dejó de } \\
\text { entrevistar a } \\
\text { varias personas } \\
\text { que no quisieron }\end{array}$ \\
\hline $\begin{array}{l}\text { Aspectos del } \\
\text { análisis } \\
\text { relacionados } \\
\text { específica- } \\
\text { mente con el } \\
\text { ARS en este } \\
\text { trabajo }\end{array}$ & $\begin{array}{c}\text { Ego-red, } \\
\text { variables, } \\
\text { multiplicidad de } \\
\text { lazos. Correla- } \\
\text { ción estadística } \\
\text { (red observada, } \\
\text { red estructural). } \\
\text { Densidad (ta- } \\
\text { maño, lazos, } \\
\text { pares, compo- } \\
\text { nentes, pasos). } \\
\text { Conectividad } \\
\text { (caminos, punto } \\
\text { de corte, distan- } \\
\text { cia, alcance). } \\
\text { Visualización de } \\
\text { la ego-red }\end{array}$ & $\begin{array}{l}\text { Indicadores } \\
\text { estructurales } \\
\text { de la red } \\
\text { (densidad, } \\
\text { centralidad, } \\
\text { centraliza- } \\
\text { ción, inter- } \\
\text { mediación, } \\
\text { cercanía) y } \\
\text { grafos de cada } \\
\text { grupo de } \\
\text { sujetos en } \\
\text { estudio }\end{array}$ & $\begin{array}{l}\text { Visualización de } \\
\text { la ego-red. } \\
\text { Devolución de la } \\
\text { visualiza-ción } \\
\text { de la red al } \\
\text { informante } \\
\text { como vía de } \\
\text { obtención de } \\
\text { nueva } \\
\text { información }\end{array}$ & $\begin{array}{l}\text { Visualización de } \\
\text { la estructura de } \\
\text { redes y relacio- } \\
\text { nes que se } \\
\text { establecen entre } \\
\text { instituciones y } \\
\text { grupos }\end{array}$ & $\begin{array}{l}\text { Composición de } \\
\text { la red. } \\
\text { Visualización de } \\
\text { redes } \\
\text { personales }\end{array}$ \\
\hline $\begin{array}{l}\text { Sofware } \\
\text { específico para } \\
\text { la representa- } \\
\text { ción de redes } \\
\text { sociales) }\end{array}$ & $\begin{array}{c}\text { Netdraw } 1 \\
\text { Ucinet }\end{array}$ & Ucinet & Egonet & - & Ucinet 6 \\
\hline Sofware (otro) & - & Spss & Egonet & - & Spss \\
\hline
\end{tabular}

Fuente: Elaboración popia. Se excluye del cuadro el trabajo de Brandes y otros (2005) para ser presentado con más detalle en el apartado siguiente. Su énfasis teórico sobre cuestiones relativas a la visualización de las redes sociales hace que los detalles metodológicos de las dos investigaciones que toma de ejemplo no sean lo suficientemente explícitos y detallados para incorporarlos en el cuadro. 
A resultas de los elementos descritos en el cuadro anterior (que sintetizan algunos elementos de los textos incluidos en este volumen), pueden ser relatados algunos de los aspectos hoy típicos en una parte importante del ARS en el ámbito internacional. Nos centramos en ellos por el carácter de introducción al volumen noveno de Redes pero cabe afirmar que una lectura detenida de los trabajos que están siendo publicados recientemente en este ámbito pueden describirse atendiendo a algunas de las pautas que se encuentran esbozadas en el cuadro previo. Me refiero, por ejemplo, a diferentes prácticas:

- Aunque existan orientaciones disciplinares predominantes, las prácticas concretas de investigación diluyen las barreras disciplinares en la medida en que una misma investigación pasa por momentos más o menos orientados hacia lo cuantitativo o cualitativo. Citando un ejemplo, en el trabajo de Teves (2005) se aprecia cómo, con una orientación antropológica, se llevan a cabo instrumentos cualitativos de recogida de información, entrevistas semiestructuradas, para posteriormente- ordenar y transformar los datos de forma que permitan un cálculo cuantitativo de correlaciones. Uno de los elementos de interés del ARS es, a mi modo de ver, precisamente esta triangulación teórica, metodológica y técnica.

- Combinar estrategias de recogida de datos clásicas (entrevista convencional, por ejemplo) con modernas (preguntas pensadas específicamente para el ARS) e insertar esto en un contexto donde los resultados obtenidos de practicar "generadores de nombres" se llevan al análisis con un software novedoso y especializado produce en ocasiones cuestiones nuevas $y$, a veces, resultados que difieren de los obtenidos con aproximaciones más convencionales. Se aportan también nuevas líneas de trabajo y enfoques dentro de algunas disciplinas, con la innovación y discusiones que ello puede comportar en el marco disciplinar concreto (véanse también los resultados del trabajo de Teves sobre este punto).

- Otra práctica cada vez más común es la combinación de diferentes programas informáticos especializados en la investigación, enriqueciéndose ésta, por ejemplo, con el aporte del software especializado en redes sociales (más moderno) a la vez que con programas más habituales y extendidos. Sería el caso de Palacio y Madariaga (2005) que combinan tanto Spss (para el cálculo del test de la t) con Ucinet para el estudio de las redes sociales. También de Gualda (2005), que articula el uso de Ucinet con la más clásica formulación de hipótesis estadísticas y cruces de variables con apoyo de Spss. Lo interesante de estas prácticas, frecuentes hoy, es que son reflejo de una mayor libertad y flexibilidad en la investigación que, a fin de enriquecer su conocimiento, emplea los instrumentos a su alcance, rompiendo "corsés" y prácticas disciplinarias más ortodoxas o extendidas (véase, por ejemplo, en Krempel y Plümer, 2003). Nuestra opinión al 
respecto de estas prácticas es favorable, en la medida en que impliquen una adaptación a su particular objeto de conocimiento.

- Respecto a este volumen, se encuentran en él trabajos que abordan la visualización y el análisis de las redes sociales con el apoyo de software especializado, sea éste Egonet (en el artículo de Aguilar), Ucinet (en los trabajos de Teves, Palacio y Madariaga y Gualda) o Visone (en Brandes y otros). Junto a éstos, el escenario actual se compone de un software más diverso donde se incluyen algunos como Pajek (http://vlado.fmf.uni-lj.si/pub/networks/pajek/); Sienna (http://stat.gamma.rug.nl/siena.html); Netminer (http://www.netminer. com/ NetMiner/home 01.jsp); u otros, que no siempre se especializan en las mismas tareas y funciones. Una referencia más detallada de este software puede rastrearse en la web del "Internacional Network for Social Network Analysis", concretamente en el apartado sobre "Computer Programs for Social Network Analysis" (http://www.insna.org/ INSNA/soft inf.html, ). Se avecina, intuimos, un futuro en el que se desarrollarán nuevos programas para el ARS, que competirán entre sí, por una parte, y que, probablemente como los actuales, se aproximarán en mayor medida a comunidades de investigadores y líneas teóricas existentes en este campo (orientaciones egocéntricas o sociocéntricas, por ejemplo).

- Puede hablarse también de la capacidad de análisis que se gana cuando la informática y sus recientes desarrollos en software hacen factible en la práctica caminos que la intuición y el trabajo "a mano" de otros investigadores ya habían sido señalados (véase sobre este particular lo que en este volumen señalan Brandes y otros -2005- en cuanto a la visualización de las redes sociales y el trabajo inicial de Moreno con los sociogramas). O el recorrido histórico descrito por Freeman (2004), cuando sitúa antes de Moreno el origen del tratamiento visual interesado en el ARS.

- Pero al mismo tiempo, se hace palpable el interés que tienen los trabajos sobre redes sociales en nuestra comprensión del mundo, independientemente de que se empleen vías más o menos próximas al uso de la tecnología informática, incluyéndose aquí trabajos que, para aportar explicaciones plausibles donde se incorpora una reflexión sobre la influencia de las redes sociales, no necesitan del uso de ninguna tecnología específica (Forni y Nardone, 2005). Recuerda esta línea de análisis a la presencia e importancia que en autores clásicos ocupaba ya la interacción social, sin que se hubiera empezado a definir un campo específico de análisis de "redes sociales".

- Algunas barreras disciplinarias se diluyen, con aportaciones en ambos sentidos. Así, por ejemplo, si en algunas investigaciones es difícil saber dónde empiezan y terminan las aportaciones de disciplinas conexas (esto es relativamente 
habitual si pensamos en las ciencias sociales y en campos afines como la sociología, la psicología social, la antropología sociocultural, etc.), en el análisis de las redes sociales (aunque no es exclusivo) se muestra también el intercambio disciplinario entre ámbitos más lejanos, de forma que podemos observar prácticas del estilo a la creación de software para aplicaciones en la investigación social (por ejemplo, el trabajo de Brandes y otros, 2005; etc); el desarrollo de algoritmos en el ámbito de la informática para lograr como meta mejorar el estudio de la conectividad de las redes sociales (Hummon y Doreian, 1990); el uso de software químico para analizar estructuras moleculares -Mage- (Molina, 2001). O la aparición de nuevas líneas de análisis que conectan y fomentan el diálogo interdisciplinario, como ocurre con el caso de la ecología y la sociología (véase el trabajo de Johnson, Borgatti, Luczkovich y Everett (2001). Son éstos ejemplos de que en el campo del ARS se muestra la reconstrucción que opera en las ciencias y que se concreta en la realización de trabajos arraigados en varias disciplinas, o hibridación disciplinaria. Sean estos trabajos realizados desde equipos monodisciplinares pero que recogen influencias pluridisciplinares, sea por el mismo trabajo conjunto de equipos interdisciplinares, que facilitan el intercambio y que juegan con una concepción más abierta de la ciencia, en la que se potencia la "hibridación de las ciencias". A nuestro juicio, las aportaciones de este tipo de operaciones de investigación son bien interesantes pues aportan luces en campos antes invisibilizados, o simplemente no considerados, además de ser un estímulo para la creatividad y, por tanto, para la gestación de óptimas condiciones para el avance en las ciencias. No sería descabellado afirmar, en este sentido, que el ámbito de las redes sociales debe en gran medida su desarrollo exponencial a esta circunstancia.

Algunos de los elementos descritos en el doble sentido de señalamiento de tendencias en el ARS y la presentación del volumen noveno, son ejemplo palmario de integración de métodos, técnicas y software de investigación a fin responder más adecuadamente a las cuestiones de investigación planteadas. Se conjugan en estos trabajos en gran medida tanto la combinación como la triangulación. El resultado viene a ser que algunos resultados de la investigación en redes sociales son, podríamos decir, de carácter "híbrido", en la medida en que una coherente articulación disciplinaria desfigura los límites disciplinarios.

\section{Practicando la visualización de las redes sociales: de procedimientos sencillos a técnicas más sofisticadas}

Aunque son varios los trabajos de este número en el que la visualización de las redes sociales ocupa un lugar especial, debe citarse el de Brandes y otros 
(2005) como el que más discute en el plano teórico sobre qué significa la visualización de las redes para el avance de este campo del saber. Esta centralidad arranca de trabajos previos de Brandes respecto a la importancia de lo visual. Por ejemplo, en Brandes, Raab y Wagner (2001) se argumenta que los resultados obtenidos no se habrían conseguido si no se hubieran llegado a visualizar, subrayando, por tanto, el interés de la visualización por sí misma, no sólo como algo meramente accesorio en la investigación. Esta misma línea de reflexión está en su trabajo publicado en este volumen, en el que, sobre la base de dos investigaciones diferentes, se defiende el valor explicativo que puede tener la visualización de las redes sociales. Visualización que tanto puede aportar un valor en la exploración de los datos, como ayudar a entenderlos o explicarlos. No obstante, es en la localización y demostración de mecanismos causales a través de la visualización donde los autores perciben más dificultades y aspectos por resolver. Habría que subrayar igualmente que la visualización de las redes sociales y personales puede jugar diferentes papeles en la investigación, entre los que se encuentran los citados anteriormente (explorar, comprender, explicar), u otros como describir o comunicar los resultados de la investigación (Freeman, 2000).

La utilidad de la visualización se ha mostrado en otras investigaciones recientes. Por ejemplo, explorando el rol de personajes políticos a través de análisis de noticias (Johnson y Krempel, 2004). Pero igualmente es interesante la aproximación organizacional de uno de los trabajos que toman como ejemplo Brandes y otros (2005). En este trabajo las mismas organizaciones sociales serían los nodos, más allá que representar relaciones interpersonales. Esta perspectiva organizacional de análisis de las redes sociales la encontramos en este volumen igualmente en Forni y Nardone (2005), aunque en este caso sin emplear un software específico para el análisis de las redes sociales.

Siguiendo con el volumen noveno, igualmente puede darse valor a la visualización en el trabajo de Teves ante el que, al descubrir la red de caminos por los que transcurre la actividad textil en el valle cachalquí salteño, puede venirnos a la cabeza la pregunta de qué pasaría en la zona si los puntos centrales en el trayecto vivieran alguna transformación relevante. En cualquier caso, se trata de ejemplos que ponen de relieve la diversidad de gráficos visuales a los que se puede dar lugar (representando palabras, personas, organizaciones, espacios geográficos...). Destáquese igualmente el valor de gráficos que, al representar, dar color y forma a los atributos, permiten visualizar más rápidamente relaciones entre las variables de interés, facilitando la constatación o negación de las hipótesis planteadas (tomemos como ejemplo los textos de Aguilar, 2005; y Palacio y Madariaga, 2005). 
El trabajo de investigación puede ser diseñado desde un principio con el fin de visualizar para lograr conocimiento (o dando un paso más: "sabiduría", en los términos en que se explica por Brandes y otros, 2005) o puede incorporarse la visualización en otros momentos, con mero carácter "ilustrativo" (a veces), o con finalidades que persigan tanto explorar como comprender y explicar. La visualización puede combinarse de diferentes formas, inclusive, en una misma investigación. Tomando ahora como ejemplo nuestro propio trabajo (Gualda, 2005a y 2005b), mostramos una de estas vías en las que la visualización se incorpora en medio de un proceso complejo de investigación. Nos referimos a una parte de nuestro estudio sobre las Necesidades de la población mayor en Berrocal y Villanueva de las Cruces: modelo de servicios y viabilidad de la implantación de un sistema sostenible de co-pago. Concretamente respecto a la influencia de las redes sociales en la calidad de vida y, específicamente para este artículo, en la autopercepción de felicidad de los de 55 y más años del municipio de Berrocal según el tamaño de sus redes personales. Se consultó sobre la red personal con apoyo de la batería de Barrera $(1980)^{3}$ a 115 mayores del municipio. En el cuestionario se incluyeron otras preguntas, entre las que estaban los rasgos sociodemográficos o la misma autopercepción de felicidad. Para este ejemplo, nos centraremos en aquellos casos en los que, cuando se les preguntó por su grado de felicidad ( $P 15$. ¿En que medida se considera una persona feliz? 0-10), respondieron de manera totalmente optimista y asignaron un "10" (dieron esta respuesta 9 personas de 115 entrevistadas). La respuesta contraria (0) sólo la aportaron 2 personas. Globalmente, y contando con los 115 entrevistados, se obtuvo un grado de felicidad media de 7,4 puntos. Sólo un 5,2\% se autoclasificó como una persona no feliz (0-3); un 20,9\% ni feliz ni no feliz (4-6) y un 73,9\%, el grupo más amplio, se clasificó como feliz (7-10).

Para explorar visualmente si el tamaño de la red personal citada nos podía dar alguna pista y estaba asociado al grado de felicidad declarado, se determinó seleccionar, por un lado, a los "más felices" (los que asignaron un 10) y, por otro, a los "menos felices" (que asignaron un 0 , un 3 o un 4, siempre una especie de representación de "suspenso" en la felicidad). Resultaron ser 18 casos, 9 por cada extremo, el de los que se autoconsideraban más y menos felices de un conjunto de 115. A partir de aquí se construyó una matriz simétrica en la que se incluyeron estos 18 casos y se añadieron a todas las personas que además fueron citadas por

\footnotetext{
${ }^{3}$ Útil para los objetivos y condicionantes de nuestra investigación, pero infravalorada por el instrumento que se empleó, que no permite obtener redes tan amplias como las que se están obteniendo a través de la metodología propuesta por McCarty $(2002,2003)$.
} 
ellos. Sólo estas 18 personas de referencia, al generar su red personal ( $A$ continuación dígame las personas con las que cuenta para...-preguntar siempre: ¿alguien más?), citaron a un total de 109 personas (entre las que hemos incluido las 18 para construir la matriz). Esto es, de los 115 entrevistados por nosotros hay 66 personas que no son citadas por ninguno de nuestros 18 casos (o egos), pero en cambio, configuran la red personal de los egos un total de 60 personas que residen en Berrocal y son menores de 55 años (hijos y amigos generalmente, no entrevistados por nosotros, que nos centramos en los de 55 y más años). Sólo se cita en esta red básica de personas a 3 que no residen en el pueblo. Se menciona a 49 que son del pueblo y han sido también entrevistados en nuestro estudio al ser de 55 y más años ${ }^{4}$. A fin de garantizar el anonimato en la muestra de resultados, se asignó aleatoriamente a cada caso (egos y alters) la denominación de "casoX".

Usando como referencia la matriz simétrica de 109 casos construída empleamos Ucinet (Borgatti, Everett and Freeman, 2002) para dibujar las relaciones personales que citaron los 18 mayores que se autodeclaraban más felices y menos felices. El resultado es haber encontrado diversos tipos de redes, algunas más simples y otras más complejas. Mostramos en los gráficos siguientes un ejemplo de varias pautas encontradas (Cuadro 2). Por una parte, encontramos sólo un caso aislado que no citó a nadie en su red (caso3). Coincide éste con una persona que asigna un 0 tanto a su percepción de felicidad como a su satisfacción ante la vida. También valora con un 4 (suspende) su confianza hacia los demás.

A pesar de habernos encontrado con más frecuencia que los que se declaran menos felices (0) citan a menos personas en su red personal (caso3 y caso96), también vemos cómo declarándose feliz (10), la red citada puede ser mínima (caso103). Marca la diferencia con respecto a otros que se declaran muy felices el bajo nivel de confianza declarado hacia los demás de esta persona (sólo de 2 , en una escala de 0-10).

Respecto a autovaloraciones más elevadas de felicidad (puntuaciones de 3 y 4 sobre un máximo de 10), se constató la tendencia a citar redes de mayor tamaño. El caso21 sería un ejemplo de esto. Este ego citó a seis personas, si bien no se localizó una red conectada con otras. En cambio, el caso72 y el caso65, que citan a un número menor de personas y que se autodeclararon con el máximo nivel de felicidad, se encuentran conectados a través del caso83, que ambos citan. Esta pauta se repite en otras ocasiones en las que se señala el máximo grado de

\footnotetext{
${ }^{4}$ Al contar con el Padrón detallado por nombre y apellidos del municipio pudo localizarse con total exactitud a los nombres generados en las entrevistas para evitar repeticiones en la matriz.
} 
felicidad y ello coincide con declaraciones de un máximo nivel de confianza hacia los demás. No debe perderse de vista que estamos describiendo las relaciones personales de mayores de 55 y más años residentes en un entorno rural.

CUADRO 2. AUTOPERCEPCIÓN DE FELICIDAD Y RED PERSONAL: ALGUNAS TIPOLOGÍAS

\begin{tabular}{|l|c|c|}
\hline No felices (0) & No feliz (3) & Feliz (10) \\
\hline casos & caso3 & \\
\hline caso96 &
\end{tabular}

Fuente: Elaboración propia a partir de Gualda (2005a). Nota: El círculo representa a los EGO, mayores felices o no felices (que citan en su red personal a otras personas). El cuadrado representa a los citados, o ALTERS, como integrantes de la red personal. Por colores, al tratarse de un estudio dirigido a la población mayor y citar ésta a personas no entrevistadas, se asignó el color rojo a todos aquellos que fueron citados, pero no entrevistados, por lo que no conocemos su grado de felicidad. En azul si se autoclasificaron como felices (asignando de 7-10 puntos). En negro, en un caso menos extremo de poca felicidad (3). En gris, si declararon felicidad 0 . Las fechas han de entenderse siempre en la dirección de la relación encontrada entre un ego (en círculo) y un alter (cuadrado), pero no al revés.

Este ejemplo sencillo pone de relieve cómo a través de la visualización de las redes, introducida en el proceso de investigación, pueden ser sugeridas nuevas cuestiones para el análisis, que podrán ser motivo de trabajos posteriores. Preguntas como, por ejemplo, ¿qué representaciones visuales se obtendrán cuando, en vez de emplear la matriz de relaciones egos-alters basada en la selección de 18 casos, los más y menos felices, se incorporen a la matriz todos los egos entrevistados (un total de 115), en una matriz donde se incorporan valores intermedios de autodeclaraciones de felicidad?, ¿Aparecerán entonces nuevas conexiones que hagan que, por ejemplo, egos aislados sean elegidos como alters por otras personas? Con este foco se ha podido apreciar que los egos menos felices tendieron a citar a menos personas en su red, del mismo modo que ocurría respecto a aquellos egos felices con un bajo nivel de confianza en los demás. ¿Podría significar esto que existe algo de ocultación de datos y se ha sobreestimado la percepción de felicidad, o es que sentirse feliz es independiente del número de personas que se citan en la red y guarda relación con otras situaciones de la vida tales a la vida social, la vida en pareja u otras?... ¿Qué diferencias encontraremos cuando se representen visualmente la red personal global, respecto a las redes existentes para aspectos específicos: material, información, etc.? Ésta y otras preguntas surgen de la simple visualización de una sencilla representación gráfica. 
También parece confirmarse que, a pesar de ciertas pautas encontradas, los factores que afectan a la autopercepción de felicidad son variopintos, como se ha destacado en otras investigaciones (Crossley y Langdridge, 2005). E igualmente se constatan pautas de interés sobre las que volver en más profundidad, como por ejemplo, varios casos en los que ocurren simultáneamente una autodeclaración de un máximo de felicidad (10), una red personal mínima o ausente y un bajo grado de confianza hacia los demás. ¿Está detrás de ese tamaño reducido de la red la confianza interpersonal, o hay otros factores adicionales?, ¿Porqué mostrándose desconfiado, se autodeclara uno tan feliz?

Este ejemplo nos sirve para argumentar que la visualización, no sólo permite resumir y mostrar a los demás los resultados de nuestro trabajo, sino igualmente sugerir nuevas líneas y ayudar con la interpretación de los datos. En estudios sociales, generalmente afectados por la participación conjunta de muchas variables, uno de los aspectos que podría quizás contribuir en mayor medida a su avance es conseguir fórmulas sencillas para la visualización de temas complejos, en los que participan múltiples variables.

$Y$ junto a esto, una de las preocupaciones que podría ser de un tipo de sociología más macro, la de cómo representar visualmente las redes de un ingente volumen de personas y que el resultado sea comprensible y analíticamente productivo. ¿Qué nuevos criterios técnicos pueden ser incorporados para lograr este tipo de representaciones?

Por otra parte, en la medida en que están temporalmente afectados, también profundizar en la visualización del paso del tiempo y cómo afecta éste en la construcción, destrucción o reconstrucción de redes sociales y personales pueden ser elementos de interés (aunque sobre este tema ya están trabajando algunos equipos y desarrollando software con estos fines, véase en www.insna.org).

Por otra parte, algunos planteamientos éticos de fondo en las Ciencias Sociales y Humanas podemos encontrarlos ligados a prácticas recientes de visualización. Me refiero al hecho de "devolver la información" obtenida al sujeto entrevistado, con objetivos que, por una parte, son éticos $y$, por otra, pueden mejorar el análisis y matizar la información obtenida. Como quiera que "una imagen vale más que mil palabras", si esta imagen es buena, permitirá discutir sobre la misma con los sujetos de la investigación (es el caso del trabajo de Aguilar en este volumen, pero también lo encontramos en otros textos recientes -McGrath y Blythe, 2004-).

La compatibilidad entre diferentes programas informáticos (por ejemplo, Atlas ti, Spss, Excel, Access, Ucinet, Egonet, etc.) permite un avance notable para el investigador en cuanto a ganancias en tiempo y en posibilidades analíticas 
respecto a lo que es viable y no en la investigación social, si bien, aún quedan mejoras por hacer en esta materia. Teniendo en cuenta que gran parte de los trabajos en Ciencias Sociales se nutren de datos textuales, una línea en la que trabajar puede ser en intentar profundizar en el tránsito y reconocimiento de archivos típicamente empleados para obtener datos textuales (hoy cada vez más, por ejemplo, archivos en .pdf), con la idea que puedan ser reconocidos y procesados directamente ( $\sin$ necesidad de transcribirlos manualmente) de cara a la visualización de los datos. Pero ésta es sólo una de muchas sugerencias que están surgiendo enfocadas hacia la implementación de mejoras en la visualización de las redes sociales.

\section{Contextos espaciotemporales y reconstrucciones teóricas del ARS}

El análisis de las redes sociales, aunque se trata de un ámbito de conocimiento que se encuentra desarrollado desigualmente en el territorio mundial, cuenta hace unas décadas con equipos de investigación que van alcanzando logros empíricos en diversas partes del mundo, en áreas de mayor y menor desarrollo socioeconómico, en ámbitos donde se dispone de mayor y menor seguridad política. Esta circunstancia, el contexto donde se arraiga el ARS, es especialmente interesante porque ha posibilitado, y lo hará en el futuro, matizar algunos planteamientos teóricos del mismo análisis de redes, en adecuación al contexto social en el que las redes sociales se despliegan. Así, por ejemplo, como se pone de manifiesto en el volumen noveno de esta Revista Redes, el planteamiento de Granovetter (1973) respecto a que la existencia de "lazos débiles" puede ser entendida como capital social de utilidad para alcanzar recursos (puentes) de los que carecemos en nuestro propio entorno personal ("lazos fuertes"), puede quedar matizado aludiendo a que éste debe revisarse en contextos muy empobrecidos en los que las personas que actúan como lazos débiles no lo hacen como "puentes" de acceso a información y recursos, por cuanto viven la misma precariedad social que los incluidos como "lazos fuertes" (sería el caso descrito de la población desplazada a que se refieren Palacio y Madariaga, 2005).

Nos recuerda esto el trabajo de Webster, Freeman y Aufdemberg (2001), cuando, a partir de dos conjuntos de datos, profundizan en la idea de que las limitaciones impuestas por los contextos sociales influencian la interacción social. Aunque en este trabajo se sugiere también que los individuos pueden de alguna forma manejar su contexto para tener menos constreñimientos. 
Curiosamente, los contextos afectan a las estrategias y pautas de interacción no sólo en el plano individual, sino igualmente en cuanto a las estrategias institucionales. De nuevo el trabajo de Forni y Nardone (2005) pone de manifiesto que en contextos de pobreza las instituciones modifican sus formas de interacción con las personas. En este caso, se utilizan recursos como la "garantía solidaria" del grupo para otorgar los microcréditos bajo el supuesto de que el grupo social minimiza los riesgos crediticios, aunque estos presupuestos, como señalan los autores, no son necesariamente cubiertos en todos los casos.

Igualmente podría decirse de la influencia del discurso oficial en Bosnia y Herzegovina $(\mathrm{BiH})$, que diferencia entre la población serbia, croata y bosníaca o musulmana, que parece estar asociada a las relaciones personales descritas en el trabajo de Aguilar (2005); y del contexto natural (recursos), que afecta a la ruta de localidades más importante de la actividad textil según la descripción de Teves (2005); o igualmente del contexto de inestabilidad política descrito en el caso de Palacio y Madariada (2005), que afecta a la situación de los desplazados.

Contextos que ponen de relieve que, a pesar de que pueda manejarse un foco de análisis que da valor a las relaciones sociales y a la interacción (en un plano más próximo al microanálisis), éstas no pueden ser desgajadas de su contexto de referencia, más ahora en un sentido macro, con el que se conjugan y en el que se despliegan y desarrollan. En este sentido, aunque analíticamente nos concentremos en la red social o personal y en las interacciones que ésta implica, una adecuada y global comprensión parece necesitar ir "más allá de" la red descrita. A la inversa, tampoco sería completa la visión de lo social, si se queda ausente la dinámica relacional. Pero ambas visiones extremas, aunque sean descritas parcialmente en algunos trabajos, pueden producir descripciones y explicaciones suficientemente plausibles para no descartarlas de nuestro panorama intelectual.

\section{Concluyendo: ¿̇hacia la consolidación del ARS como espacio disciplinario híbrido?}

Como ocurre en otros temas de interés para la investigación social, habría que decir que el análisis de las redes sociales incorpora en su seno elementos del clásico debate en ciencias sociales respecto a si empezó primero "el huevo o la gallina", o dicho de otra manera, si la realidad está más conformada por aspectos macro o por aspectos micro. Nuestra argumentación al respecto ha sido conducida más hacia un camino de múltiples direcciones, asumiendo la pluralidad que caracteriza a los objetos de conocimiento habituales de las ciencias sociales y, en este caso, a los mismos objetos (y sujetos) de conocimiento del ARS. Como 
correlato necesario, por coherencia, el despliegue metodológico y técnico debe ir en consonancia con las características de lo que queremos conocer (Beltrán, 2000). Pluralidad, por otra parte, que no debe ser entendida en el sentido de algo "ilimitado" o "infinito", sino más bien, como la asunción de que puede haber variabilidad en nuestros objetos y sujetos de conocimiento, así como en las aproximaciones teóricas, métodos y técnicas con los que nos aproximamos.

Uno de los elementos que integran nuestra realidad es precisamente la interacción, vínculo o relación que se produce entre actores individuales y colectivos y en este punto parece tener su razón de ser el ARS. Pero hemos argumentado que ello es compatible con el análisis de la situación contextual en que se produce la relación social, entendiendo de manera generosa el contexto o marco (social, discursivo, cultural, geográfico, económico,...). De hecho, los trabajos que se han presentado en este volumen no son ajenos a dicho contexto y enriquecen su análisis con su conocimiento. No nos encontramos preparados, a las alturas del desarrollo científico, para resolver genéricamente si juegan un papel más prominente unos elementos u otros. En lo que sí puede abundarse, ahora refiriéndome al más estricto campo del ARS, es en el valor del análisis de las redes sociales y personales para nuestra comprensión de algunos fenómenos. A veces este enfoque es productivo por cuanto plantea nuevas preguntas, otras porque ayuda a redefinir conceptos, en ocasiones porque aporta matices, etc. $y$, globalmente, porque produce conocimiento. $Y$, como suele ocurrir en el ámbito científico, porque más que producir conclusiones definitivas, deja las puertas "entreabiertas" a indagaciones posteriores. Algunas de ellas tan importantes como analizar el cambio social a través de las redes sociales y personales, profundizar en el estudio de fenómenos de carácter "meso" e incluso "macro", en ir algo más allá del experimentalismo o las simulaciones, pero sin negar su utilidad en algunos momentos, desarrollar nuevos enfoques, métodos y técnicas de estudio de las redes sociales, etc.

Volviendo al tema de fondo en este trabajo, habría que preguntarse ahora con Hummon y Carley (2002) en qué medida el ARS, aunque es evidente el estrecho vínculo que tiene con el análisis estructural de la interacción social, ha superado o puede superar dichos límites. Una segunda cuestión, sobre la que se discute en Freeman (2004) es sobre si el proceso histórico de configuración de este campo disciplinar ha culminado en una nueva disciplina. A nuestro modo de ver, parece haberse ido configurando (sin poder darse el proceso por concluido) un espacio disciplinar híbrido, como producto de un camino histórico irregular, a través del que a veces se habló de "redes sociales" pero sin nombrarlas de esa manera (nos remontamos, por ejemplo, al XIX e inicios del XX) y en otras ocasiones nos 
encontramos con contribuciones aisladas, o con disputas disciplinarias, hasta llegar épocas más próximas en las que se incrementa notablemente la productividad científica en el campo del ARS. Un espacio híbrido en el sentido de ser espacio producido por elementos de distinta naturaleza, con alusión directa ahora al resultado del intercambio disciplinar o científico.

Por otra parte, aunque es innegable el que hay más temas o asuntos que se abordan hoy desde la perspectiva del ARS, así como hemos visto incrementarse las publicaciones, eventos y comunidades de especialistas en este campo, cabe preguntarse si el proceso de configuración como disciplina está cerrado. Sugeríamos hace un momento que nos parecía un proceso no concluido.

Estoy entendiendo ahora la idea de "campo disciplinar" como algo más amplio y complejo que la idea de "campo de investigación" y cabría preguntarse si el proceso de reconocimiento e institucionalización del ARS ha culminado hasta el punto de que desde otras disciplinas se haya reconocido la especificidad. Pensando, por ejemplo, en España, podríamos hablar de la, parece, incipiente consolidación del campo de las "Ciencias del Trabajo", que se ha traducido en la formulación de una titulación académicamente reglada, diversas facultades en el territorio español y ya, alguna que otra promoción de licenciados en "Ciencias del Trabajo", en el futuro, algunos de ellos doctores e investigadores. ¿Ha llegado el ARS a consolidarse de forma equivalente? En estos momentos no, pero quizás podría ocurrir a corto plazo. ¿O quizás se siga percibiendo este campo como "accesorio" a otras disciplinas que se interesan por ellas?

Lo que sí parece claro es que habría materia, y muy variada, sobre la que configurar un currículo formativo en la enseñanza universitaria tomando como eje el ARS. Inclusive podrían aportarse especializaciones y orientaciones, más hacia ciencias sociales o humanas y más hacia ciencias técnicas, por ejemplo. Lo mismo ocurre con respecto a las agendas de investigación, aunque en este caso, nos parece que el campo de investigación está más consolidado. Sobre qué futuro vendrá, intelectualmente nos parece muy productivo pensar en la riqueza que aporta la "hibridación" disciplinaria, apostando por ir "más allá de" las Ciencias Sociales y Humanas. Sobre la manera concreta de llegar, no sólo al intercambio interdisciplinario, sino también al reconocimiento y consolidación en un contexto de disciplinas asentadas, los caminos concretos pueden ser diversos, pues ya ni siquiera es necesaria la acumulación de especialistas en el mismo entorno físico para lograrlo, al poderse concretar espacios de formación e investigación virtuales. Como enfatiza Kadushin (2005), en su presentación del libro de Freeman (2004): "There remains a large world for grounded social network ideas to conquer". 


\section{BIBLIOGRAFÍA}

Aguilar, C. (2005). "Visualización de redes personales en Sarajevo". Redes. Revista hispana para el análisis de redes sociales. Vol.9\#4, Dic.2005, http://revistaredes.rediris.es, pp. 1- 14.

Barrera, M. (1980). "Method for the Assessment of social support. Network in community survey research". Connections (3), pp.8-13.

Beltrán, M. (2000). Perspectivas sociales y conocimiento. Anthropos-UAM, Barcelona.

Borgatti, S.P., Everett, M.G. and Freeman, L.C. (2002). Ucinet for Windows: Software for Social Network Analysis. Harvard, MA: Analytic Technologies.

Brandes, U.; Kenis, P. y Raab, J.: "Explanation Through Network Visualization". Redes. Revista hispana para el análisis de redes sociales. Vol.9\#4, Dic.2005, http://revista-redes.rediris.es, pp. 1-27.

Brandes, U.; Raab, J. y Wagner, D. (2001). "Exploratory Network Visualization: Simultaneous Display of Actor Status and Connections". Journal of Social Structure. Vol.2, núm.4 (http://www.cmu.edu/joss/).

Crossley, A. y Langdridge, D. (2005). "Perceived sources of Happiness: a network Analysis". Journal of Happiness Studies, 6, pp.107-135.

Forni, P. y Nardone, M. (2005). "Grupos solidarios de microcrédito y redes sociales: sus implicancias en la generación de capital social en barrios del Gran Buenos Aires". REDES. Revista hispana para el análisis de redes sociales. Vol.9\#5, Dic.2005, http://revista-redes.rediris.es, pp. 1- 25.

Freeman, L.C. (2000). "Visualizing Social Networks". Journal of Social Structure. Vol.1, núm.1, (http://www.cmu.edu/joss/).

Freeman, L.C. (2004). The Development of Social Network Analysis: A Study in the Sociology of Science. Vancouver, Canada: Booksurge Publishing.

Granovetter, M. (1973). "The strength of weak ties". American Journal of Sociology, 78, pp.1360-1380.

Gualda, E. (2005a). "Redes de apoyo social y personal en Berrocal y Villanueva de las Cruces. En Gualda, E. (Dir.): Necesidades de la población mayor en Berrocal y Villanueva de las Cruces: modelo de servicios y viabilidad de la implantación de un sistema sostenible de co-pago. Informe Avance. Tomo I. En prensa.

Gualda, E. (2005b). "Redes sociales y personales y su implicación en los estados de ánimo y percepciones de felicidad de personas mayores. Estudio de casos en Berrocal y Villanueva de las Cruces (Huelva)". I International Workshop de la Red Hispana para el Análisis de Redes Sociales, 7-9 septiembre, Sevilla. http://revistaredes.rediris.es/webredes/red tematica/Sevilla2 
Hummon, N.P. y Carley, K. (1993). "Social networks as normal science". Social Networks. Vol.15, 1, marzo, pp.71-106.

Hummon, N.P. y Doreian, P. (1990): "Computational methods for social network analysis". Social Networks, vol. 12, 4, pp.273-288.

Johnson, J.C. y Krempel, L. (2004). "Network Visualization: The "Bush Team" in Reuters News Ticker 9/11-11/15/01". Journal of Social Structure. Vol.5 (http://www.cmu.edu/joss/).

Johnson, J.C.; Borgatti, S.P.; Luczkovich, J.J.; Everett, M.G. (2001). "Network Role Analysis in the Study of Food Webs: An Application of Regular Role Coloration". Journal of Social Structure. Vol.2, núm.3 (http://www.cmu.edu/joss/).

Kadushin, C. (2005): "Reseña del libro de Freeman, L.C. (2004). The Development of Social Network Analysis: A Study in the Sociology of Science". En Journal of Social Structure. http://www.cmu.edu/joss/.

Krempel, L. y Plümper, T. (2003). "Exploring the Dynamics of International Trade by Combining the Comparative Advantages of Multivariate Statistics and Network Visualization". Journal of Social Structure. Vol.4, núm.1. http://www.cmu.edu/joss/.

McCarty, C. (2002). "Structure in Personal Networks", Journal of Social Structure, vol.3, núm.1 (http://www.cmu.edu/joss/).

McCarty, C. (2003). Egonet. Personal Network Software. http://survey.bebr.ufl. edu/EgoNet/.

McGrath, C. y Blythe, J. (2004). "Do You See What I Want You to See? The Effects of Motion and Spatial Layout on Viewers' Perceptions of Graphical Structures". Journal of Social Structure. Vol.5, núm.2 (http://www.cmu.edu/ joss/).

Molina, J.L. (2001). El análisis de redes socials. Una introducción. Bellaterra, Barcelona.

Palacio Sañudo, J. y Madariaga Orozco, C.A (2005). "Redes sociales personales y calidad de vida en personas desplazadas por violencia política: el caso de Barranquilla (Colombia)". Redes. Revista hispana para el análisis de redes sociales. Vol.9\#3, Dic.2005, http://revista-redes.rediris.es, pp. 1- 27.

Teves, L. (2005). "Análisis de Redes sociales y actividades económicas en las comunidades de Molinos". Redes. Revista hispana para el análisis de redes sociales. Vol.9\#2, Dic.2005, http://revista-redes.rediris.es, pp. 1- 25.

Webster, C.M.; Freeman, L.C. y Aufdemberg, C.G. (2001). "The Impact of Social Context on Interaction Patterns". Journal of Social Structure. Vo.2, núm.1. http://www.cmu.edu/joss/. 